\title{
Reperfusion Normalizes Motor Activation Patterns in Large-Vessel Disease
}

\author{
Mohamad Chmayssani, MD, ${ }^{1}$ Ronald M. Lazar, PhD, ${ }^{1}$ Joy Hirsch, PhD, ${ }^{2}$ and Randolph S. Marshall, MS, $\mathrm{MD}^{1}$
}

\begin{abstract}
Objective: Hemodynamic impairment in one hemisphere has been shown to trigger ipsilateral motor activation in the opposite hemisphere on functional imaging. We hypothesized that reversing the hypoperfusion would normalize the motor activation pattern.

Methods: We studied four patients with high-grade stenosis and impaired vasomotor reactivity (VMR) but no stroke. Functional magnetic resonance imaging motor activation pattern before and after VMR normalization was compared with seven healthy control subjects scanned at an interval of 3 months using voxel-wise statistical parametric maps and region of interest analysis. Subjects performed a repetitive hand closure task in synchrony with $1 \mathrm{~Hz}$ metronome tone. We used repeated-measures analysis of variance to compute the interaction between group (patients/control subjects) and time by obtaining the average blood oxygen level dependent signal of three motor regions of interest in each hemisphere.

Results: Two patients normalized their VMR after spontaneous resolution of dissection, and two after revascularization procedures. Both voxel-wise statistical maps and region of interest analysis showed that VMR normalization was associated in each case with a reduction in the atypical activation in the hemisphere opposite to the previously hypoperfused hemisphere $(p<$ 0.001).

Interpretation: In the presence of a physiological stressor such as hypoperfusion, the brain is capable of dynamic functional reorganization to the opposite hemisphere that is reversible when normal blood flow is restored. These findings are important to our understanding of the clinical consequences of hemodynamic failure and the role of the ipsilateral hemisphere in maintaining normal neurological function.
\end{abstract}

Ann Neurol 2009;65:203-208

The central nervous system has a unique ability to dynamically reorganize in response to pathological stressors such as stroke. ${ }^{1}$ It has been shown with functional imaging that whereas healthy subjects generally activate contralateral motor cortex while performing a simple hand motor task, the same task after injury generates additional activity in the ipsilateral hemisphere, ${ }^{2}$ which then reduces over time. ${ }^{3,4}$ We previously expanded the range of attributable cerebral triggers associated with emergence of ipsilateral motor activation, demonstrating the existence of ipsilateral task-related activation in patients with large-vessel disease and impaired vasomotor reactivity (VMR) despite the absence of stroke $e^{5}$ or transient ischemic attack. ${ }^{6}$ These findings suggested that a state of unilaterally impaired cerebral hemodynamics is sufficient to alter the motor activity pattern. A question that remained to be answered was whether a reversal of the hypoperfusion would result in normalization of the ipsilateral motor-related activity in the other hemisphere. A return to a canonical pattern would suggest that neurons in the hypoperfused hemisphere existed in a dysfunctional but viable state capable of regaining function if physiological conditions are restored. On the other hand, detecting no change in the ipsilateral activation would suggest that hemodynamic impairment induced a subclinical neuronal injury that disrupted irreversibly the primary circuits of the brain, resulting in a lasting recruitment of alternative brain regions to share the neuronal burden of normal neurological function. We investigated these two alternative possibilities by examining the brain activation patterns in patients with unilateral cerebral hemodynamic impairment whose hemodynamic failure subsequently reversed. We hypothesized that hemodynamic normalization, as measured by VMR using transcranial Doppler (TCD) with $5 \% \mathrm{CO}_{2}$ inhalation, would reverse the increased motor-related functional
From the ${ }^{1}$ Department of Neurology, Stroke Division, Columbia University Medical Center, New York; and ${ }^{2}$ Department of Radiology, Program of Imaging and Cognitive Sciences, Columbia University Medical Center, New York, NY.

Address correspondence to Dr Marshall, Department of Neurology, Columbia University Medical Center, 710 West 168th Street, New York, NY 10032. E-mail: rsm2@columbia.edu

Potential conflict of interest: Nothing to report.
Additional Supporting Information may be found in the online version of this article.

Received Jun 5, 2008, and in revised form Aug 8. Accepted for publication Sep 12, 2008.

Published online in Wiley InterScience (www.interscience.wiley.com). DOI: 10.1002 /ana.21554 
magnetic resonance imaging (fMRI) signal in the opposite hemisphere.

\section{Subjects and Methods Subjects}

Four patients with large-vessel disease, impaired VMR, and no history of stroke or transient ischemic attack were compared with seven healthy control subjects. Patients were consecutively drawn from cerebrovascular patients evaluated by the neurovascular service who met eligibility criteria. Patient 1 was a 48 -year-old woman with a left carotid artery dissection. She had spontaneous recanalization 3 months later, with a concomitant normalization of VMR. Patient 2 was a 53-year-old man with right middle cerebral artery (MCA) high-grade stenosis who had spontaneous normalization of VMR when measured 18 months later. Patient 3, a 58-year-old man, underwent stenting of a high-grade left internal carotid stenosis, restoring normal VMR at 3 months. Patient 4, a 78-year-old man with right internal carotid artery high-grade stenosis, also underwent stenting that restored normal VMR as measured by TCD on his third postoperative day. Normal VMR was still present 2 months later at the time the patient underwent a follow-up fMRI scan. Seven healthy control subjects, aged $58 \pm 9$ years, had normal neurological examination, no evidence for focal lesions on fluid-attenuated inversion recovery or diffusion-weighted imaging magnetic resonance images, and normal carotid and TCD studies. All participants gave written informed consent for the study using an institutional review board-approved, Health Insurance Portability and Accountability Act (HIPAA)-compliant protocol. (For additional clinical information, see supplementary data.)

\section{Vasomotor Reactivity Measurement}

All patients underwent cerebral VMR testing to determine cerebral hemodynamic status. Bilateral TCD monitoring (Pioneer TC 4040; Nicolet Biomedical, Madison, WI) of the MCAs was performed at an insonation depth of 50 to $56 \mathrm{~mm}$, as described previously. ${ }^{7}$ After 2 minutes of baseline measurement, subjects breathed $5 \% \mathrm{CO}_{2}$ (carbigene) via face mask for 2 minutes. VMR was calculated as percentage increase in ipsilateral MCA mean flow velocity per millimeter of mercury partial pressure of $\mathrm{CO}_{2}$. The contralateral VMR was measured as a control, expected always to be in the reference range. "Normal" VMR was defined as an increase in MCA mean flow velocity of at least $2.0 \% / \mathrm{mm} \mathrm{Hg}$ partial pressure of $\mathrm{CO}_{2}$, corresponding to two standard deviations less than the mean of control data from a previous study. ${ }^{7}$

\section{Imaging}

During $\mathrm{fMRI}$, subjects performed a repetitive hand closure task in synchrony with $1 \mathrm{~Hz}$ metronome click in three 20 second blocks alternating with rest. Imaging was performed on a General Electric (GE) $1.5 \mathrm{~T}$ magnet, $128 \times 128 \mathrm{ma}-$ trix, $19 \mathrm{~cm}^{2}$ field of view, slice thickness $=4.5 \mathrm{~mm} / 0$ skip, $\mathrm{TR}=4,000, \mathrm{TE}=60$, flip angle $=60$ degrees, 25 slices, functional voxel dimensions $=1.5 \times 1.5 \times 4.5 \mathrm{~mm}$. Image volumes were coregistered, motion and slice timingcorrected, subjected to high-pass filter, normalized to Ta- lairach template, and smoothed with an $8 \mathrm{~mm}$ Gaussian kernel. The first three volumes of each run were excluded for signal stabilization. For all patients, motion correction (three transposition planes, three directions of rotation) was well within the functional voxel dimensions. VMR and fMRI measurements were performed on the same day.

\section{Voxel-wise Analysis}

Images were flipped to make the "right" the hypoperfused hemisphere and the "left" the normally perfused hemisphere. Therefore, the left hand, contralateral to the hypoperfused hemisphere, was designated as the "affected hand," and the right hand in patients and control subjects was designated as the "unaffected hand."

A fixed-effects group analysis compared the blood oxygen level dependent (BOLD) activity in the four patients at Time 1 (baseline) and Time 2 (after normalization of VMR) with the BOLD activity of the control groups at Time 1 and Time 2, evaluating for a group $\times$ time interaction. The interaction contrast was therefore represented as (P2-P1) - (C2-C1), where P1 and P2, and C1 and C2 represented task-related fMRI data at Time 1 and Time 2, from patients and control subjects, respectively (null hypothesis: $\mathrm{P} 2-\mathrm{P} 1=\mathrm{C} 2-\mathrm{C} 1$; that is, no difference in change in activity over time between groups). We were particularly interested in change in the ipsilateral motor regions where previous studies had shown increased activity in response to hypoperfusion in the opposite hemisphere. ${ }^{5,8}$ All contrasts were assessed at a threshold of $\mathrm{t}=3.0$, corresponding to $p=0.0027$ uncorrected, a common threshold for fMRI studies of this type. A formal statistical test using regions of interest (ROIs) was performed to confirm our findings (see later). To exclude the possibility that a group effect was driven by one or two patients, we examined the Time $2-$ Time 1 contrast in each patient individually.

\section{Region of Interest Analysis}

To quantitatively test our hypothesis that the atypical activation in the ipsilateral motor cortical areas decreased as a result of VMR normalization, we performed an independent ROI analysis among all subjects over two time points by computing average BOLD signal intensity ( $\beta$ value) in the primary sensorimotor cortex (M1S1), lateral premotor cortex, and supplementary motor area (SMA). ROIs were drawn using each subject's T1-structural MRI scan as a template to maximize the specificity of the ROIs, following the anatomical definition of these areas that Fink and colleagues ${ }^{9}$ described. ROI creation was blinded to the activation pattern to avoid bias. The $\beta$ of the three ROIs in each hemisphere at each time point was averaged, then entered into a repeated-measures analysis of variance to look for interaction between groups (patients vs control subjects) and time. Two computations were performed: the laterality index $\left(\beta_{\text {contra }}-\beta_{\text {ipsi }}\right) /\left(\beta_{\text {contra }}+\beta_{\text {ipsi }}\right)$ and absolute $\beta$ value of the ipsilateral hemisphere alone.

\section{Results}

Voxel-wise Statistical Parametric Map

All patients and control subjects had normal neurological function at the time of testing. No mirror move- 


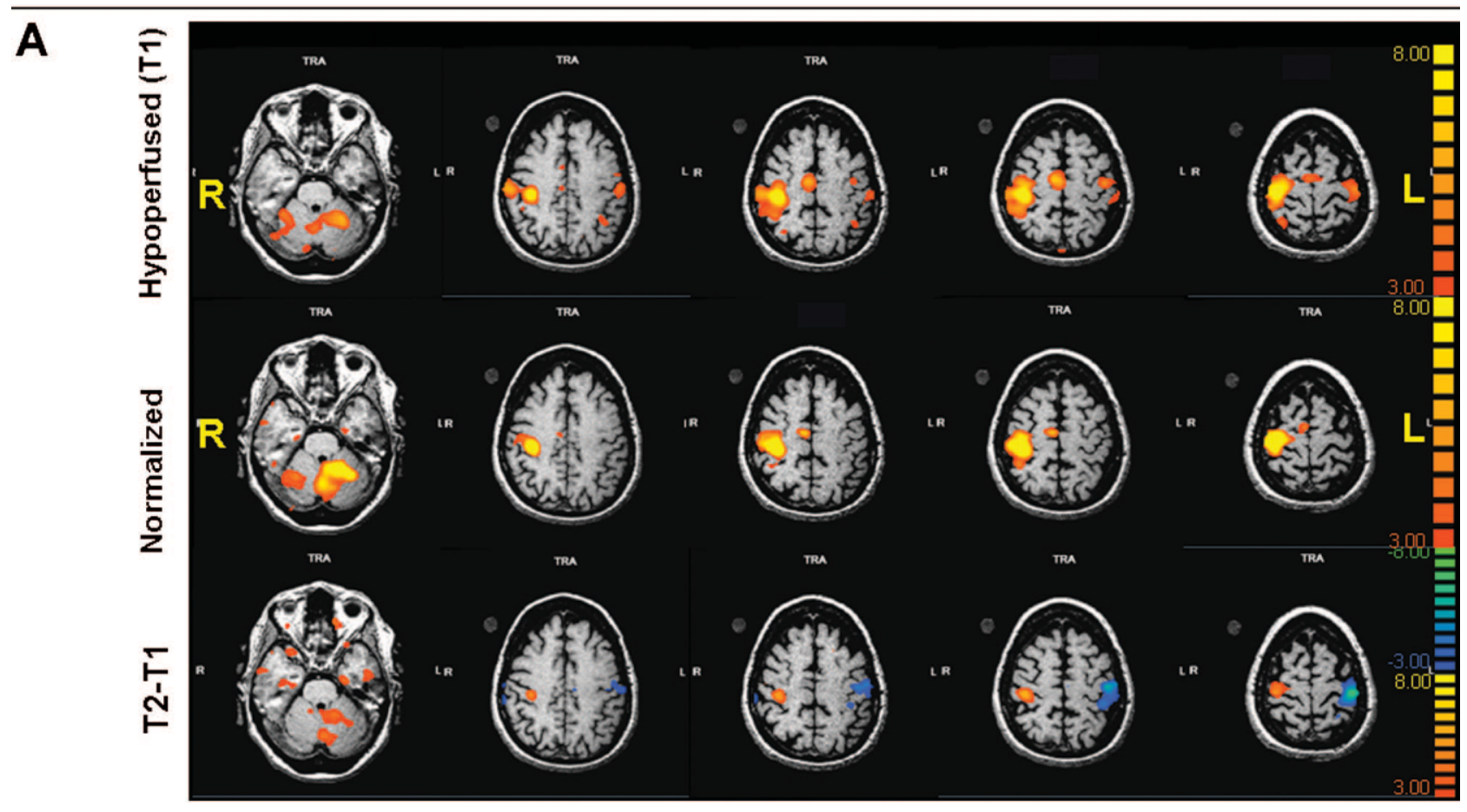

B
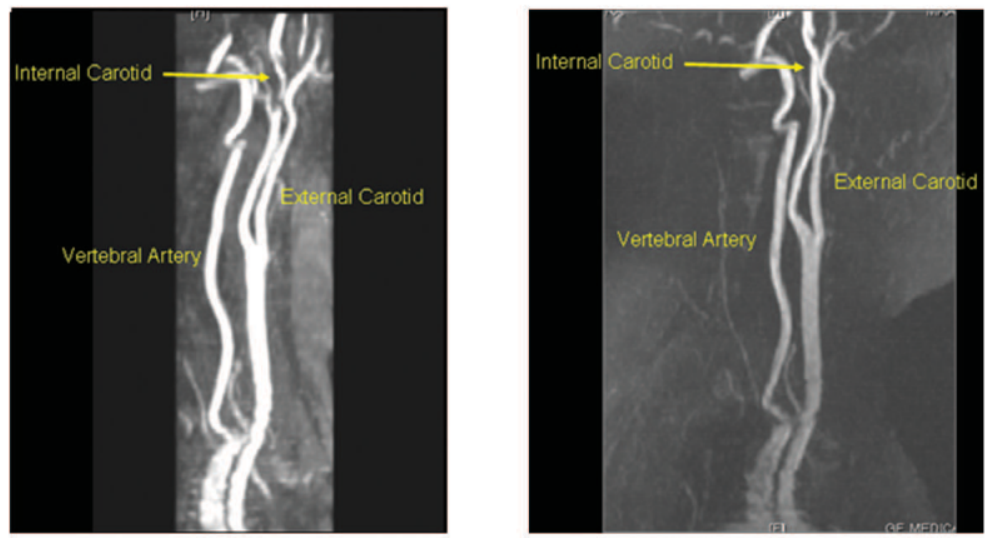

Fig 1. (A) Axial activation maps of Patient 1 at baseline hypoperfusion state (row 1), after normalization of hemodynamics (row 2) and using the contrast Time 1 minus Time 2 (T2-T1; $t<3$; $\mathrm{p}<0.0027)$. At hypoperfused baseline, "affected hand" movement yielded activation in ipsilateral and contralateral hemispheres, whereas after normalization of blood flow, activation is confined to contralateral hemisphere. In row 3, using the contrast, T2-T1, a significant reduction in blood oxygen level dependent (BOLD) signal (blue) in ipsilateral hemisphere is seen. Color bars to the right of each condition indicate the range of activation $T$ values. (B) Left internal carotid artery dissection in producing abnormal vasomotor reactivity (VMR) spontaneous recanalization 3 months later associated with normal VMR is shown.

ments were detected visually during brain scans. As expected, control subject hand movement and the "unaffected hand" of patients was associated with activation predominantly in contralateral $M_{1} S_{1}$, premotor and supplementary motor area. In contrast, "affected hand" movements in patients with impaired VMR activated additional motor areas in the ipsilateral hemisphere, confirming previous work. ${ }^{5,8}$ Figure 1 illustrates in Patient 1 the decrease in BOLD signal associated with recanaliza- tion of her carotid dissection and consequent normalization of VMR. The T2-T1 contrast in each of the other three patients, as well as group data comparing patients with control subjects, produced similar results (Fig 2).

Region of Interest Analysis

For the average BOLD signal in the three motor region ROIs, there was significant group $\times$ time interaction driven by an increase in laterality index in patients 


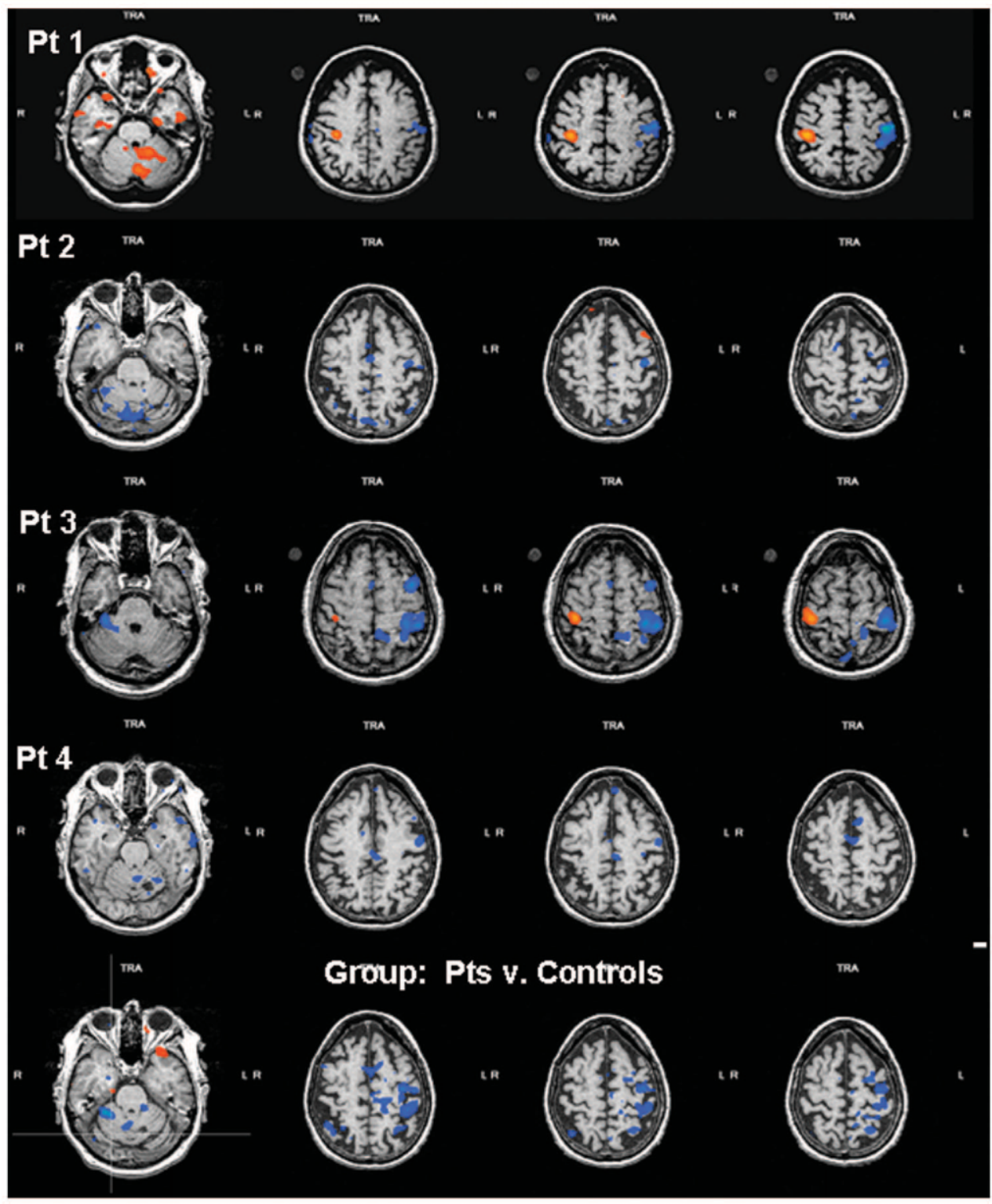

Fig 2. Axial activation maps of Patients 1 to 4 (rows 1-4) using the contrast Time 2 minus Time 1 (T2-T1) showing a significant reduction in BOLD signal (blue) in ipsilateral hemisphere (Left) with the movement of left hand, "affected hand." Group analysis (row 5): patients versus control subjects using a fixed-effects model with (P2-P1) - (C2-C1), where P1 and P2 refer to the blood oxygen level dependent (BOLD) signal at the two time points for patients, and C1 and C2 the same for control subjects, demonstrating reduction in BOLD signal in ipsilateral $M_{1} S_{1 c}$, dorsal pre-motor, and supplementary motor area (SMA) (see Fig 3, row 5), after normalization of vasomotor reactivity $(t<3$; $\mathrm{p}<0.0027)$.

compared with control subjects, indicating a shift of activity toward the contralateral hemisphere $(p<0.01$; Fig 3A; see Supplementary Table 2). We found a significant decrease in BOLD intensity in patients as compared with control subjects in the ipsilateral hemisphere after VMR normalization, confirming that the interaction was contributed significantly to by changes in the ipsilateral hemisphere $(p<0.001$ and Supplementary Table 2). These findings were reproducible in each of the four patients (see Fig 3B). Also consistent with our prior work, the BOLD activation in the three motor ROIs in the hypoperfused hemisphere at baseline was slightly but not significantly reduced compared with control subjects (1.37 vs $2.46 ; p=0.06$ ), suggesting that there was preserved neurovascular coupling despite the hemispheral hypoperfusion.

\section{Discussion}

In this prospective, proof-of-principle study, we extend our previous findings of abnormal hemodynamics inducing ipsilateral motor activation ${ }^{6}$ by demonstrating that removal of the trigger of hemodynamic failure is 
A

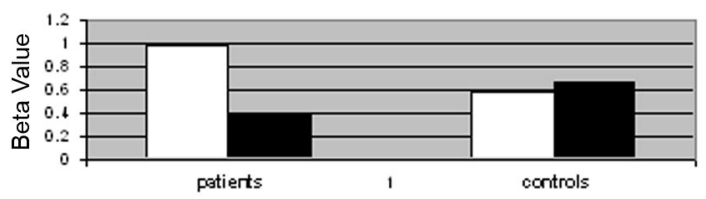

B

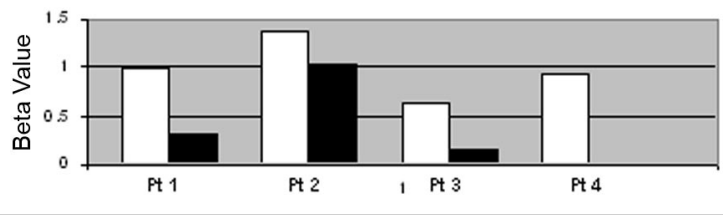

Fig 3. (A) Average blood oxygen level dependent (BOLD) signal ( $\beta$ value) of ipsilateral hemisphere. Time (baseline with impaired hemodynamics vs 3-month follow-up with normalized hemodynamics) by group (patients vs control subjects) interaction, showing that patients had a significant decrease in $\%$ BOLD signal over time, whereas no significant change occurred over time in control subjects. Values at each time point are BOLD signal averaged across three regions of interest. (B) Average BOLD signal ( $\beta$ value) in the ipsilateral hemisphere over time in each individual patient. Consistent with the group analysis, \% BOLD signal in the ipsilateral hemisphere decreased in each of the patients after normalization of vasomotor reactivity. White bars designate baseline state; black bars designate the postrevascularization state.

associated with restoration of a typical, predominantly contralateral motor activation pattern. Our findings suggest that impaired VMR is not only sufficient to produce an alteration in motor networks, but is a reversible trigger as well.

The mechanism of the phenomenon we observed has yet to be determined. The presence of activity in the opposite hemisphere may be the result of crosshemispheral disinhibition as has been demonstrated after experimental ${ }^{10}$ and clinical ${ }^{11}$ stroke. The return to a typical balance of contralateral-ipsilateral activity in response to revascularization suggests that neurons in the hypoperfused hemisphere may exist in a dysfunctional but viable state that can be reversed if physiological conditions are restored. Such a state has been difficult to demonstrate in humans, although reversal of cognitive dysfunction has been reported after restoration of normal cerebral hemodynamics with heart transplant, ${ }^{12}$ carotid endarterectomy, ${ }^{13}$ and extracranialintracranial bypass. ${ }^{14}$ Evidence at both cellular and clinical levels for such a process comes from chronically ischemic myocardium that resumes function by restoring coronary blood flow. ${ }^{15-17}$

A similar state of reversible dysfunction has also been described in neurons in rats subjected to chronic ischemia. Neurons were shown to reduce their energy needs when diminished energy substrates are accessible. ${ }^{18,19}$ The likelihood that neurons would regain function with restoration of blood flow is thought to depend on the acuity of ischemia, ${ }^{20}$ duration of isch- emia, ${ }^{17}$ volume of ischemia, ${ }^{21}$ and degree of hypoperfusion. ${ }^{19}$ For our patients, it may be that the severity, duration, and volume of hypoperfusion were great enough to impair normal neuronal activity but not great enough to cause irreversible damage. The relative acuity with which the presence of ipsilateral BOLD activation was identified in Patient 1, 6 weeks after carotid dissection, suggests that the process may happen fairly quickly. Reorganization in experimental ${ }^{10}$ stroke has been shown to appear within minutes, clinically as early as 24 hours. $^{3}$

An alternative explanation for our findings might be that the carotid occlusion caused a relative shunting of blood flow to the opposite hemisphere, resulting in higher BOLD signals there; then when the vessel recanalized, the blood flow was redistributed away from that side, resulting in a reduction in BOLD. This scenario is unlikely because a normally functioning hemisphere should have intact autoregulation, maintaining normal cerebral blood flow regardless of whether the opposite hemisphere has a reduced flow. Furthermore, quantitative cerebral blood flow measurements in carotid occlusion show no increased blood flow in the opposite hemisphere. $^{22}$

Limitations of our study include a small sample size. Our group results reached statistical significance, however, and more importantly, results were demonstrated in each individual patient. Another concern is an alternative explanation for the change in laterality index in which a reduction in BOLD activity in the hypoperfused hemisphere could be caused by neurovascular uncoupling, ${ }^{23}$ which then could have been reversed when the hemisphere was reperfused. Although we found a mildly reduced BOLD signal in the hypoperfused hemisphere in our cohort, it is the presence of activity in the opposite hemisphere that constituted evidence for reorganization, and the demonstrated reduction in activation with revascularization that indicated its reversibility.

Finally, our results have important implications for understanding the role of the ipsilateral hemisphere in sustaining normal neurological function. An absence of a subtle motor dysfunction before revascularization could not be completely ruled out because we based our assessment of normality on the patients' lack of symptoms and a normal neurological examination. Our study is unique in showing presence and reversal of increased ipsilateral activation in the absence of symptoms, in contrast with most studies of brain reorganization in cerebrovascular patients in which ipsilateral motor activation is associated with persistent neurological dysfunction. ${ }^{4,24,25}$ The idea of silent, brain reorganization has precedent in the neuroscience literature, in describing neuroplasticity in the setting of chronic brain tumors ${ }^{26}$ and arteriovenous malformations, ${ }^{27}$ as well as in descriptions of unconscious skill learning in 
healthy subjects. ${ }^{28}$ Further study is needed to elucidate the physiological mechanisms underlying the reversible recruitment of the ipsilateral hemisphere in the absence of a structural brain lesion or a symptomatic state.

This work was supported by the NINDS (5R01HD043249) and the Levine Research Fund.

\section{Acknowledgment}

We thank B. Minzer for his technical assistance.

\section{References}

1. Weiller C, Chollet F, Friston KJ, et al. Functional reorganization of the brain in recovery from striatocapsular infarction in man. Ann Neurol 1992;31:463-472.

2. Johansen-Berg H, Rushworth MF, Bogdanovic MD, et al. The role of ipsilateral premotor cortex in hand movement after stroke. Proc Natl Acad Sci U S A 2002;99:14518-14523.

3. Marshall RS, Perera GM, Lazar RM, et al. Evolution of cortical activation during recovery from corticospinal tract infarction. Stroke 2000;31:656-661.

4. Ward NS, Brown MM, Thompson AJ, Frackowiak RS. Neural correlates of motor recovery after stroke: a longitudinal fMRI study. Brain 2003;126:2476-2496.

5. Krakauer JW, Radoeva PD, Zarahn E, et al. Hypoperfusion without stroke alters motor activation in the opposite hemisphere. Ann Neurol 2004;56:796-802.

6. Marshall RS, Krakauer JW, Matejovsky T, et al. Hemodynamic impairment as a stimulus for functional brain reorganization. J Cereb Blood Flow Metab 2006;26:1256-1262.

7. Marshall RS, Rundek T, Sproule DM, et al. Monitoring of cerebral vasodilatory capacity with transcranial Doppler carbon dioxide inhalation in patients with severe carotid artery disease. Stroke 2003;34:945-949.

8. Marshall RS, Krakauer JW, Radoeva PD, et al. Hemispheric hemodynamic impairment in the absence of stroke induces fMRI activation in the opposite hemisphere. Neurology 2004; 62:A541.

9. Fink GR, Frackowiak RS, Pietrzyk U, Passingham RE. Multiple nonprimary motor areas in the human cortex. J Neurophysiol 1997;77:2164-2174.

10. Strens LH, Fogelson N, Shanahan P, et al. The ipsilateral human motor cortex can functionally compensate for acute contralateral motor cortex dysfunction. Curr Biol 2003;13: $1201-1205$.

11. Gerloff C, Bushara K, Sailer A, et al. Multimodal imaging of brain reorganization in motor areas of the contralesional hemisphere of well recovered patients after capsular stroke. Brain 2006;129:791-808.
12. Deshields TL, McDonough EM, Mannen RK, Miller LW. Psychological and cognitive status before and after heart transplantation. Gen Hosp Psychiatry 1996;18:62S-69S.

13. Fearn SJ, Hutchinson S, Riding G, et al. Carotid endarterectomy improves cognitive function in patients with exhausted cerebrovascular reserve. Eur J Vasc Endovasc Surg 2003;26: $529-536$

14. Sasoh M, Ogasawara K, Kuroda K, et al. Effects of EC-IC bypass surgery on cognitive impairment in patients with hemodynamic cerebral ischemia. Surg Neurol 2003;59:455-463.

15. Braunwald E, Rutherford JD. Reversible ischemic left ventricular dysfunction: evidence for the "hibernating myocardium." J Am Coll Cardiol 1986;8:1467-1470.

16. Marban E. Myocardial stunning and hibernation. The physiology behind the colloquialisms. Circulation 1991;83:681-688.

17. Rahimtoola SH. From coronary artery disease to heart failure: role of the hibernating myocardium. Am J Cardiol 1995;75: $16 \mathrm{E}-22 \mathrm{E}$.

18. de la Torre JC, Fortin T, Park GA, et al. Brain blood flow restoration 'rescues' chronically damaged rat CA1 neurons. Brain Res 1993;623:6-15.

19. de la Torre JC, Saunders J, Fortin T, et al. Return of ATP/PCr and EEG after $75 \mathrm{~min}$ of global brain ischemia. Brain Res 1991;542:71-76.

20. Marshall RS, Lazar RM, Pile-Spellman J, et al. Recovery of brain function during induced cerebral hypoperfusion. Brain 2001;124:1208-1217.

21. Ohta $H$, Nishikawa $H$, Kimura $H$, et al. Chronic cerebral hypoperfusion by permanent internal carotid ligation produces learning impairment without brain damage in rats. Neuroscience 1997;79:1039-1050.

22. Grubb RL Jr, Derdeyn CP, Fritsch SM, et al. Importance of hemodynamic factors in the prognosis of symptomatic carotid occlusion. Jama 1998;280:1055-1060.

23. Powers WJ, Fox PT, Raichle ME. The effect of carotid artery disease on the cerebrovascular response to physiologic stimulation. Neurology 1988;38:1475-1478.

24. Calautti C, Leroy F, Guincestre JY, et al. Sequential activation brain mapping after subcortical stroke: changes in hemispheric balance and recovery. Neuroreport 2001;12:3883-3886.

25. Feydy A, Carlier R, Roby-Brami A, et al. Longitudinal study of motor recovery after stroke: recruitment and focusing of brain activation. Stroke 2002;33:1610-1617.

26. Petrovich NM, Holodny AI, Brennan CW, Gutin PH. Isolated translocation of Wernicke's area to the right hemisphere in a 62-year-man with a temporo-parietal glioma. AJNR Am J Neuroradiol 2004;25:130-133.

27. Lazar RM, Marshall RS, Pile-Spellman J, et al. Anterior translocation of language in patients with left cerebral arteriovenous malformation. Neurology 1997;49:802-808.

28. Willingham DB, Salidis J, Gabrieli JD. Direct comparison of neural systems mediating conscious and unconscious skill learning. J Neurophysiol 2002;88:1451-1460. 